\title{
The Cayley Trick, lifting subdivisions and the Bohne-Dress theorem on zonotopal tilings
}

Received February 18, 1999 / final version received January 25, 2000

Published online May 22, 2000 - (c) Springer-Verlag \& EMS 2000

Abstract. In 1994, Sturmfels gave a polyhedral version of the Cayley Trick of elimination theory: he established an order-preserving bijection between the posets of coherent mixed subdivisions of a Minkowski sum $\mathcal{A}_{1}+\cdots+\mathcal{A}_{r}$ of point configurations and of coherent polyhedral subdivisions of the associated Cayley embedding $\mathcal{C}\left(\mathcal{A}_{1}, \ldots, \mathcal{A}_{r}\right)$. In this paper we extend this correspondence in a natural way to cover also non-coherent subdivisions. As an application, we show that the Cayley Trick combined with results of Santos on subdivisions of Lawrence polytopes provides a new independent proof of the Bohne-Dress theorem on zonotopal tilings. This application uses a combinatorial characterization of lifting subdivisions, also originally proved by Santos.

\section{Introduction}

The investigations in this paper are motivated from several directions. Our point of departure is the polyhedral version of the Cayley Trick of elimination theory given by Sturmfels in [21, Sect. 5]. The Cayley Trick is originally a method to rewrite a certain resultant of a polynomial system as a discriminant of one single polynomial with additional variables [9, pp. 103ff. and Chap. 9, Proposition 1.7]. Its applications are in the area of sparse elimination theory and computation of mixed volumes $[7,10,11,13$, $14,23]$.

Mixed subdivisions of the Minkowski sum of a family $\mathcal{A}_{1}, \ldots, \mathcal{A}_{r} \subset$ $\mathbb{R}^{d}$ of polytopes were introduced in $[11,14,21]$. The polyhedral Cayley Trick of Sturmfels says that coherent mixed polyhedral subdivisions of

B. Huber: Mathematical Sciences Research Institute, 1000 Centennial Drive, Berkeley, CA 94720-5070, USA, e-mail: birk@ isc.tamu.edu

J. Rambau: Konrad-Zuse-Zentrum für Informationstechnik Berlin, Takustrasse 7, 14195 Berlin, Germany, e-mail: rambau@zib.de

F. Santos: Depto. de Matemáticas, Estadística y Computación, Universidad de Cantabria, 39005 Santander, Spain, e-mail: santos@matesco.unican.es

Work of F. Santos partially supported by grant PB97-0358 of Spanish Dirección General de Enseñanza Superior e Investigación Científica. 
the Minkowski sum of $\mathcal{A}_{1}, \ldots, \mathcal{A}_{r} \subset \mathbb{R}^{d}$ are in one-to-one refinementpreserving correspondence to coherent polyhedral subdivisions of their Cayley embedding $\mathcal{C}\left(\mathcal{A}_{1}, \ldots, \mathcal{A}_{r}\right) \subset \mathbb{R}^{r-1} \times \mathbb{R}^{d}$. (For definitions of this and the following see Sect. 2.) More precisely, it establishes a strong isomorphism between certain fiber polytopes. In Theorem 3.1, we extend this isomorphism to an isomorphism between the refinement posets of all induced subdivisions, no matter whether coherent or not. This extension needs a more combinatorial approach than the one used in [21]. We carry it out in Sect. 3 after introducing the relevant concepts in Sect. 2.

Our second motivation is that there are applications of the Cayley trick in specific cases which are of intrinsic interest. The most striking one is the Bohne-Dress theorem [5] (see also [6,18,24]) about zonotopal tilings, to which we devote Sect. 4. Other applications of the Cayley trick to triangulations of hypercubes and of products of simplices will appear in [20].

A zonotope is the affine projection of a hypercube, or equivalently, a Minkowski sum of segments. A zonotopal tiling is a subdivision induced by this projection (i.e., a subdivision into smaller zonotopes in certain conditions, see for example [24]). The Bohne-Dress theorem states that there is a one-to-one correspondence between the zonotopal tilings of a zonotope $Z$ and the single-element lifts of the oriented matroid $\mathcal{M}(Z)$ associated to $Z$. Our version of the Cayley trick, in turn, tells us that zonotopal tilings of $Z$ are in one-to-one correspondence with polyhedral subdivisions of its Cayley embedding, which in this case is a Lawrence polytope. (Lawrence polytopes have been studied mostly in connection to oriented matroid theory, see [1,6, 24], but their property of being Cayley embeddings of segments has never been pointed out before.) To close the loop, polyhedral subdivisions of a Lawrence polytope were shown to correspond to single-element lifts of the oriented matroid by Santos [19], via the concept of lifting subdivisions introduced in [6, Sect. 9.6]. We include a new and shorter proof of this last equivalence in the realizable case (Proposition 4.4). It is based on a geometric characterization of lifting subdivisions, also contained in [19]. In this way, this paper contains a complete (modulo Santos' characterization of lifting subdivisions) new proof of the Bohne-Dress theorem (Theorem 4.3). It turns out that of the three equivalences in Theorem 4.3, the most transparent is the one given by the Cayley trick, which is exhibited in this paper for the first time.

Our final motivation concerns functorial properties of subdivision posets. Given an affine map between polytopes, can one draw conclusions about the induced map between the corresponding posets of polyhedral subdivisions? For example, the intersection of a subdivision with an affine subspace yields again a subdivision of the intersection polytope. In fact, it turns out that the isomorphism given by the Cayley Trick is exactly a map of this type. We 
think it would be of interest to investigate such maps in a more general framework (even if they do not produce isomorphisms), in relation to the so-called generalized Baues problem for polyhedral subdivisions (see [16, 17] for information on this problem).

\section{Preliminaries}

\subsection{Subdivisions of point configurations}

By a point configuration $\mathcal{A}$ in $\mathbb{R}^{d}$ we mean a finite labeled subset of $\mathbb{R}^{d}$. We allow $\mathcal{A}$ to have repeated points which are distinguished by their labels. The convex hull $\operatorname{conv}(\mathcal{A})$ of $\mathcal{A}$ is a polytope.

A face of a subconfiguration $B \subseteq \mathcal{A}$ is a subconfiguration $F^{\omega} \subseteq B$ consisting of all the points on which some linear functional $\omega \in\left(\overline{\mathbb{R}}^{d}\right)^{*}$ takes its minimum over $\mathcal{A}$. Given two subconfigurations $B_{1}$ and $B_{2}$ of $\mathcal{A}$ we say that they intersect properly if the following two conditions are satisfied:

- $B_{1} \cap B_{2}$ is a face of both $B_{1}$ and $B_{2}$;

- $\operatorname{conv}\left(B_{1}\right) \cap \operatorname{conv}\left(B_{2}\right)=\operatorname{conv}\left(B_{1} \cap B_{2}\right)$.

A subconfiguration of $\mathcal{A}$ is said to be full-dimensional if it affinely spans $\mathbb{R}^{d}$. In that case we call it a cell. It is simplicial if it is an affinely independent configuration. Following [3] and [9, Sect. 7.2] we say that a collection $S$ of cells of $\mathcal{A}$ is a (polyhedral) subdivision of $\mathcal{A}$ if the elements of $S$ intersect pairwise properly and $\operatorname{cover} \operatorname{conv}(\mathcal{A})$ in the sense that

$$
\cup_{B \in S} \operatorname{conv}(B)=\operatorname{conv}(\mathcal{A}) \text {. }
$$

Cells that share a common facet are adjacent. The set of subdivisions of $\mathcal{A}$ is partially ordered by the refinement relation

$$
S_{1} \leq S_{2} \quad: \Longleftrightarrow \quad \forall B_{1} \in S_{1}, \exists B_{2} \in S_{2}: B_{1} \subset B_{2} .
$$

The poset of subdivisions of $\mathcal{A}$ has a unique maximal element which is the trivial subdivision $\{\mathcal{A}\}$. The minimal elements are the subdivisions all of whose cells are simplicial, which are called triangulations of $\mathcal{A}$.

The following characterization has already been proved for triangulations by de Loera et al. in [8]. (It is a consequence of parts (i) and (ii) of their Theorem 1.1.) Here we include a proof for subdivisions, whose final part follows the proof of their Theorem 3.2.

Lemma 2.1. Let $\mathcal{A}$ be a point configuration. Let $S$ be a collection of cells of $\mathcal{A}$. Then, $S$ is a subdivision if and only if the following conditions are satisfied: 
(i) There is a point in $\operatorname{conv}(\mathcal{A})$ that is contained in the convex hull of exactly one cell of $S$.

(ii) For every $B \in S$ and for every facet $F$ of $B$, either $F$ lies in a facet of $\operatorname{conv}(\mathcal{A})$ or there is another $B^{\prime} \in S$ of which $F$ is a facet. We say in this case that $B$ and $B^{\prime}$ are adjacent.

(iii) Any two adjacent cells in S lie in opposite halfspaces with respect to their common facet.

Proof. If $S$ is a subdivision, it is easy to verify that it satisfies (i), (ii), and (iii). Conversely, if $S$ satisfies (i), (ii) and (iii) then refining each cell in $S$ with its placing triangulation (with respect to any ordering of $\mathcal{A}$ fixed in advance) we get a collection $T$ of simplices which still satisfies (i), (ii) and (iii). By the results in [8] $T$ is a triangulation. Using this it is easy to prove that $S$ is a subdivision.

\subsection{Induced subdivisions}

Now let $P \subset \mathbb{R}^{p}$ be a polytope, and let $\pi: \mathbb{R}^{p} \rightarrow \mathbb{R}^{d}$ be a linear projection map. We can consider the point configuration $\mathcal{A}$ arising from the projection of the vertex set of $P$. An element in $\mathcal{A}$ is labeled by the vertex of $P$ of which it is considered to be the image. In other words, $\pi$ induces a bijection from the vertex set of $P$ into $\mathcal{A}$, even if different vertices of $P$ have the same projection.

A subdivision $S$ of $\mathcal{A}$ is said to be $\pi$-induced if every cell of $S$ is the projection of the vertex set of a face of $P$. With these conditions, $S$ contains the same information as the collection of faces of $P$ whose vertex sets are in $S$. In this sense one can say that a $\pi$-induced subdivision of $\mathcal{A}$ is a polyhedral subdivision whose cells are projections of faces of $P$. (This statement is not very accurate; see $[15,16,24]$ for an accurate definition of $\pi$-induced subdivisions in terms of faces of $P$.)

Every non-zero linear functional $\phi \in\left(\mathbb{R}^{p}\right)^{*}$ defines a $\pi$-induced subdivision $S_{\phi}$ as follows: $\phi$ gives a factorization of $\pi$ into a map $(\pi, \phi)$ : $\mathbb{R}^{p} \rightarrow \mathbb{R}^{d} \times \mathbb{R}$ and the map $\rho: \mathbb{R}^{d} \times \mathbb{R} \rightarrow \mathbb{R}^{d}$ which forgets the last coordinate. For any element $a \in \mathcal{A}$ let $a_{P}$ denote the unique vertex of $P$ of which it is considered to be the image by $\pi$. For any face $F$ of the $(d+1)$-dimensional polytope $(\pi, \phi)(P)$ we denote by $\mathcal{A}_{F}$ the collection of points $\mathcal{A}_{F}:=\left\{a \in \mathcal{A}:(\pi, \phi)\left(a_{P}\right) \in F\right\}$. A face $F$ of $(\pi, \phi)(P)$ is called lower if its exterior normal cone contains a vector whose last coordinate is negative. With this notation, $S_{\phi}:=\left\{\mathcal{A}_{F} \subset \mathcal{A}: F\right.$ is a lower face of $(\pi, \phi)(P)\}$ is a $\pi$-induced subdivision of $\mathcal{A}$. The subdivision $S_{\phi}$ is called the $\pi$-coherent subdivision of $\mathcal{A}$ induced by $\phi$, and a $\pi$-induced subdivision is called $\pi$-coherent if it equals $S_{\phi}$ for some $\phi$. 
Said in a more compact form, a subset $B \subset \mathcal{A}$ is a cell of $S_{\phi}$ if and only if there is a linear functional $\phi^{\prime}: \mathbb{R}^{d} \rightarrow \mathbb{R}$ such that $B$ is the subset of $\mathcal{A}$ where $\phi^{\prime} \circ \pi+\phi$ takes its minimum value. (For example, $S_{\phi}$ is the trivial subdivision if and only if $\phi$ factors by $\pi$.)

Definition 2.2 (Fiber Polytope). The poset of $\pi$-induced subdivisions excluding the trivial one is denoted by $\omega(P, \pi)$. Its minimal elements are the subdivisions for which every cell comes from a $\operatorname{dim}(\mathcal{A})$-dimensional face of $P$. They are called tight $\pi$-induced subdivisions. The subposet of $\pi$-coherent subdivisions is denoted by $\omega_{\mathrm{coh}}(P, \pi)$. It is isomorphic to the face lattice of a certain polytope of dimension $\operatorname{dim}(P)-\operatorname{dim}(\mathcal{A})$, called the fiber polytope $\Sigma(P, \pi)$.

See $[2,24]$ for more information on $\pi$-induced subdivisions and fiber polytopes.

\subsection{Weighted Minkowski sums. Mixed subdivisions}

Let $\mathcal{A}_{i}:=\left\{a_{i}^{(1)}, \ldots, a_{i}^{\left(m_{i}\right)}\right\}$ be point configurations in $\mathbb{R}^{d}$, with $i=1, \ldots, r$.

Their Minkowski sum $\sum_{i=1}^{r} \mathcal{A}_{i}$ is defined to be the set of all points which can be expressed as a sum of a point from each $\mathcal{A}_{i}$, i.e.,

$$
\sum_{i=1}^{r} \mathcal{A}_{i}:=\left\{a_{1}+\cdots+a_{r}: a_{i} \in \mathcal{A}_{i}\right\} .
$$

A vector $\lambda=\left(\lambda_{1}, \ldots, \lambda_{r}\right)$ in $\mathbb{R}^{r}$ with $\sum_{i=1}^{r} \lambda_{i}=1$ and $0<\lambda_{1}, \ldots, \lambda_{r}<1$ is a weight vector. For a weight vector $\lambda$ the weighted Minkowski sum is defined by

$$
\sum_{i=1}^{r} \lambda_{i} \mathcal{A}_{i}:=\left\{\lambda_{1} a_{1}+\cdots+\lambda_{r} a_{r}: a_{i} \in \mathcal{A}_{i}\right\} .
$$

The configuration $\sum_{i=1}^{r} \lambda_{i} \mathcal{A}_{i}$ has $\prod_{i=1}^{r} m_{i}$ points, some perhaps repeated.

A cell (i.e., full-dimensional subset) $B \subset \sum_{i=1}^{r} \lambda_{i} \mathcal{A}_{i}$ will be called a Minkowski cell if $B=\lambda_{1} B_{1}+\cdots+\lambda_{r} B_{r}$ for some non-empty subsets $B_{i} \subset \mathcal{A}_{i}, i=1, \ldots, r$. A mixed subdivision of the weighted Minkowski sum of $\mathcal{A}_{1}, \ldots, \mathcal{A}_{r}$ is a subdivision of the configuration $\sum_{i=1}^{r} \lambda_{i} \mathcal{A}_{i}$ whose faces are all Minkowski cells. (There is not complete agreement in the literature concerning this definition. See Remark 2.5.) A Minkowski cell is called fine if it does not properly contain any other Minkowski cell. A mixed subdivision is fine if all its faces are fine.

We can consider the cartesian product of point configurations as a Minkowski sum where all the point configurations lie in complementary affine subspaces. This leads to the following natural projection. 
Definition 2.3 (Weighted Minkowski Projection). Let $\mathcal{A}_{1}, \ldots, \mathcal{A}_{r}$ be point configurations in $\mathbb{R}^{d}$, and let $P_{1}, \ldots, P_{r}$ be polytopes in $\mathbb{R}^{p_{1}}, \ldots, \mathbb{R}^{p_{r}}$, resp., the vertex sets of which affinely project to $\mathcal{A}_{1}, \ldots, \mathcal{A}_{r}$ via

$$
\mathcal{P}_{i}:=\operatorname{vert}\left(P_{i}\right) \stackrel{\pi_{i}}{\rightarrow} \mathcal{A}_{i}, \quad 1 \leq i \leq r .
$$

Moreover, let $\lambda=\left(\lambda_{1}, \ldots, \lambda_{r}\right)$ be a weight vector. We define

$\lambda \Pi_{M}:=\lambda_{1} \pi_{1}+\cdots+\lambda_{r} \pi_{r}:\left\{\begin{aligned} \mathcal{P}_{1} \times \cdots \times \mathcal{P}_{r} & \rightarrow \lambda_{1} \mathcal{A}_{1}+\cdots+\lambda_{r} \mathcal{A}_{r}, \\ \left(p_{1}, \ldots, p_{r}\right) & \mapsto \lambda_{1} \pi_{1}\left(p_{1}\right)+\cdots+\lambda_{r} \pi_{r}\left(p_{r}\right) ;\end{aligned}\right.$

The projection $\lambda \Pi_{M}$ is specially interesting if the polytopes $P_{i}$ involved are simplices. The proof of the following fact is just a check of definitions.

Lemma 2.4. Let $\lambda \Pi_{M}: \mathcal{P}_{1} \times \cdots \times \mathcal{P}_{r} \rightarrow \lambda_{1} \mathcal{A}_{1}+\cdots+\lambda_{r} \mathcal{A}_{r}$ be a weighted Minkowski projection, as in Definition 2.3, and suppose that the the polytopes $P_{i}$ are all simplices. Then, a subdivision of $\lambda_{1} \mathcal{A}_{1}+\cdots+\lambda_{r} \mathcal{A}_{r}$ is (fine) mixed if and only if it is (tight) $\lambda \Pi_{M}$-induced.

Remark 2.5. There is some confusion in the literature concerning the definition of mixed subdivisions of the Minkowski sum $\sum_{i=1}^{r} \mathcal{A}_{i}$ of the family of point configurations $\left\{\mathcal{A}_{1}, \ldots, \mathcal{A}_{r}\right\}$. First of all, in most of the literature it is assumed that the number of configurations equals the dimension of the ambient space (i.e., $d=r$ ) because this is the case in the applications to zero-dimensional polynomial systems. However, the geometric proofs involved work the same without this assumption.

Pedersen and Sturmfels [14, p. 380] defined mixed subdivisions to be the subdivisions $\Pi_{M}$-induced by the projection $\Pi_{M}: \mathcal{P}_{1} \times \cdots \times \mathcal{P}_{r} \rightarrow \mathcal{A}_{1}+$ $\cdots+\mathcal{A}_{r}$ of our Lemma 2.4. Sturmfels [21, p. 213] defined coherent mixed subdivisions as the ones which are $\Pi_{M}$-coherent. This is the same as we do. However, for the applications it is interesting to pose the following additional property: that in every cell $B=B_{1}+\cdots+B_{r}$ of the subdivision the different $B_{i}$ 's lie in complementary subspaces. (This assumption allows to compute the mixed volume of $\mathcal{A}_{1}+\cdots+\mathcal{A}_{r}$ by summing up the volumes of some cells of the subdivision.) It seems that Pedersen and Sturmfels [14] implicitly assume that all mixed subdivisions have this property, since they say (p. 380) "the mixed volume ... is the sum of volumes of the parallelotopes in $\Delta$ ". In [21] the additional property is explicitly mentioned and said to hold for all fine mixed subdivisions (which are called tight there). In other literature the property is taken as part of the definition of mixed subdivision $[11,13]$; $\Pi_{M}$-induced subdivisions without this property are just called subdivisions of the $r$-tuple $\left(\mathcal{A}_{1}, \ldots, \mathcal{A}_{r}\right)$.

Finally, there seems to be agreement to call tight subdivisions the minimal elements in the poset of subdivisions induced by a projection in general $[2,16,17,24]$ and fine mixed those for the particular case of mixed 
subdivisions [11,13], with the exception of [21] mentioned above. We have chosen to follow this convention.

\subsection{The Cayley embedding}

We call the Cayley embedding of $\mathcal{A}_{1}, \ldots, \mathcal{A}_{r}$ the following point configuration in $\mathbb{R}^{r-1} \times \mathbb{R}^{d}$. Let $e_{1}, \ldots, e_{r}$ be a fixed affine basis in $\mathbb{R}^{r-1}$ and $\mu_{i}: \mathbb{R}^{d} \rightarrow \mathbb{R}^{r-1} \times \mathbb{R}^{d}$ be the affine inclusion given by $\mu_{i}(x)=\left(e_{i}, x\right)$. Then we define

$$
\mathcal{C}\left(\mathcal{A}_{1}, \ldots, \mathcal{A}_{r}\right):=\cup_{i=1}^{r} \mu_{i}\left(\mathcal{A}_{i}\right) .
$$

The Cayley embedding of point configurations from complementary affine subspaces equals the join product of the point configurations. (For the purpose of this paper we can define the join product $\mathcal{P}_{1} * \cdots * \mathcal{P}_{r}$ of several point configurations with $\mathcal{P}_{i} \subset \mathbb{R}^{p_{i}}$ to be their Cayley embedding $\mathcal{C}\left(\mathcal{P}_{1}, \ldots, \mathcal{P}_{r}\right) \subset \mathbb{R}^{r-1} \times \mathbb{R}^{p_{1}} \times \cdots \times \mathbb{R}^{p_{r}}$.) Hence, we have the following natural projection.

Definition 2.6 (Cayley Projection). Let $\mathcal{A}_{1}, \ldots, \mathcal{A}_{r}$ be point configurations in $\mathbb{R}^{d}$, and let $P_{1}, \ldots, P_{r}$ be polytopes in $\mathbb{R}^{p_{1}}, \ldots, \mathbb{R}^{p_{r}}$, resp., the vertex sets of which affinely project to $\mathcal{A}_{1}, \ldots, \mathcal{A}_{r}$ via

$$
\mathcal{P}_{i}:=\operatorname{vert}\left(P_{i}\right) \stackrel{\pi_{i}}{\rightarrow} \mathcal{A}_{i}, \quad 1 \leq i \leq r .
$$

Define

$$
\Pi_{C}:=\mathcal{C}\left(\pi_{1}, \ldots, \pi_{r}\right):\left\{\begin{aligned}
\mathcal{P}_{1} * \cdots * \mathcal{P}_{r} & \rightarrow \mathcal{C}\left(\mathcal{A}_{1}, \ldots, \mathcal{A}_{r}\right), \\
\left(e_{i}, p_{i}\right) & \mapsto\left(e_{i}, \pi_{i}\left(p_{i}\right)\right) .
\end{aligned}\right.
$$

Again, the following lemma is obvious since a join of simplices is a simplex.

Lemma 2.7. If $\mathcal{P}_{i}$ is a simplex for all $1 \leq i \leq r$ then every subdivision of $\mathcal{C}\left(\mathcal{A}_{1}, \ldots, \mathcal{A}_{r}\right)$ is $\Pi_{C}$ induced.

\section{The Cayley Trick}

In this section we state and prove the Cayley Trick for induced subdivisions.

Theorem 3.1 (The Cayley Trick for Induced Subdivisions). Let $\mathcal{A}_{1}, \ldots$, $\mathcal{A}_{r}$ be point configurations in $\mathbb{R}^{d}$. Moreover, let $P_{1}, \ldots, P_{r}$ be polytopes in $\mathbb{R}^{p_{1}}, \ldots, \mathbb{R}^{p_{r}}$, resp., the vertex sets of which affinely project to $\mathcal{A}_{1}, \ldots, \mathcal{A}_{r}$ via

$$
\mathcal{P}_{i}:=\operatorname{vert}\left(P_{i}\right) \stackrel{\pi_{i}}{\rightarrow} \mathcal{A}_{i}, \quad 1 \leq i \leq r
$$


Then for all weight vectors $\lambda=\lambda_{1}, \ldots, \lambda_{r}$ there are the following isomorphisms of posets:

$$
\begin{aligned}
\omega\left(\mathcal{P}_{1} \times \ldots \times \mathcal{P}_{r}, \lambda_{1} \pi_{1}+\ldots+\lambda_{r} \pi_{r}\right) & \cong \omega\left(\mathcal{P}_{1} * \cdots * \mathcal{P}_{r}, \mathcal{C}\left(\pi_{1}, \ldots, \pi_{r}\right)\right) \\
\omega_{\mathrm{coh}}\left(\mathcal{P}_{1} \times \ldots \times \mathcal{P}_{r}, \lambda_{1} \pi_{1}+\ldots+\lambda_{r} \pi_{r}\right) & \cong \omega_{\mathrm{coh}}\left(\mathcal{P}_{1} * \cdots * \mathcal{P}_{r}, \mathcal{C}\left(\pi_{1}, \ldots, \pi_{r}\right)\right)
\end{aligned}
$$

The second of the two equivalences above follows from [21, Theorem 5.1] and is stated only for completeness. The structure of the proof of the first one is as follows: first, we represent the Minkowski sum as a section of the Cayley embedding, then we define an explicit order-preserving map that carries the isomorphism. Finally, we show that the canonical inverse construction is well-defined and order-preserving. A "guide line" of the proof is indicated in Fig. 1.

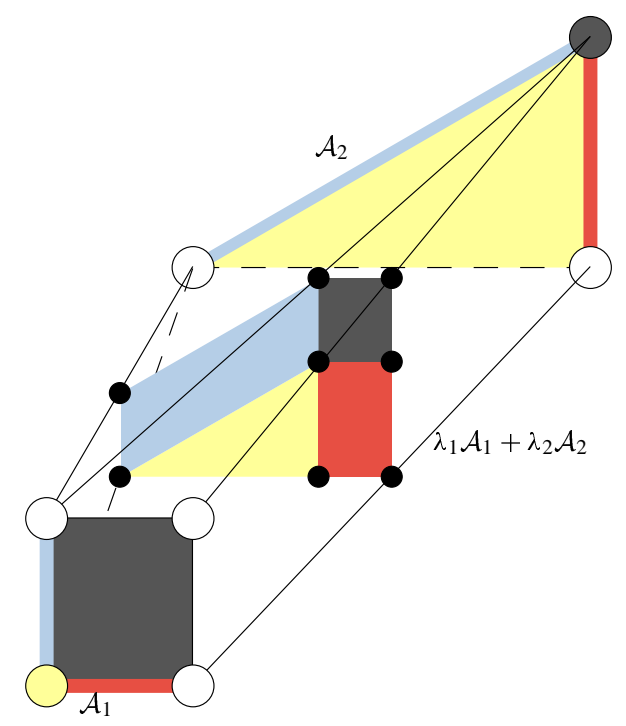

Fig. 1. "One-picture-proof of the Cayley Trick": The picture depicts the geometric interpretation of the correspondence in the Cayley Trick: intersecting the Cayley embedding with the affine subspace $W(\lambda)$ yields the Minkowski sum weighted by $\lambda$. Since all cells in a subdivision of the Cayley embedding are also Cayley embeddings this correspondence carries over to cells in subdivisions

Lemma 3.2. Let $\mathcal{A}_{1}, \ldots, \mathcal{A}_{r} \subset \mathbb{R}^{d}$ be point configurations. Moreover, let $\lambda=\left(\lambda_{1}, \ldots, \lambda_{r}\right)$ be a weight vector. (Recall this implies that $\lambda_{i}>0 \forall i$ and $\sum_{i=1}^{r} \lambda_{i}=1$.) Moreover, let $W(\lambda):=\left\{\lambda_{1} e_{1}+\cdots+\lambda_{r} e_{r}\right\} \times \mathbb{R}^{d} \subset \mathbb{R}^{r-1} \times \mathbb{R}^{d}$.

Then the scaled Minkowski sum $\lambda_{1} \mathcal{A}_{1}+\cdots+\lambda_{r} \mathcal{A}_{r} \subset \mathbb{R}^{d}$ has the following representation as a section of the Cayley embedding $\mathcal{C}\left(\mathcal{A}_{1}, \ldots, \mathcal{A}_{r}\right)$ 
in $\mathbb{R}^{r-1} \times \mathbb{R}^{d}$ :

$$
\begin{aligned}
\lambda_{1} \mathcal{A}_{1}+\ldots+ & \lambda_{r} \mathcal{A}_{r} \cong \mathcal{C}\left(\mathcal{A}_{1}, \ldots, \mathcal{A}_{r}\right) \wedge W(\lambda) \\
:= & \left\{\operatorname{conv}\left\{\left(e_{1}, a_{1}\right), \ldots,\left(e_{r}, a_{r}\right)\right\} \cap W(\lambda):\right. \\
& \left.\left(e_{1}, a_{1}\right), \ldots,\left(e_{r}, a_{r}\right) \in \mathcal{C}\left(\mathcal{A}_{1}, \ldots, \mathcal{A}_{r}\right)\right\},
\end{aligned}
$$

Moreover, $F$ is a facet of $\lambda_{1} \mathcal{A}_{1}+\cdots+\lambda_{r} \mathcal{A}_{r}$ if and only if it is of the form $F=F^{\prime} \wedge W(\lambda)$ for a facet $F^{\prime}$ of $\mathcal{C}\left(\mathcal{A}_{1}, \ldots, \mathcal{A}_{r}\right)$ containing at least one point $\left(e_{i}, a_{i}\right)$ for all $1 \leq i \leq r$.

Remark 3.3. On the level of convex hulls the above representation for the Minkowski sum polytope is nothing else but the ordinary intersection of the Cayley embedding polytope with the affine subspace $W(\lambda)$. We need the slightly more complicated version for point configurations stated above because in convex hulls - as subsets of a Euclidean space - we cannot keep track of multiple points.

Proof of Lemma 3.2. Define $q_{e}(\lambda):=\lambda_{1} e_{1}+\cdots+\lambda_{r} e_{r} \in \mathbb{R}^{r-1}$, so that

$$
W(\lambda)=\left\{q_{e}(\lambda)\right\} \times \mathbb{R}^{d} .
$$

Analogously, for any sequence $a=\left(a_{1}, \ldots, a_{r}\right)$ of points with $a_{i} \in \mathcal{A}_{i}$ we set $q_{a}(\lambda):=\lambda_{1} a_{1}+\cdots+\lambda_{r} a_{r} \in \mathbb{R}^{d}$. Then the intersection point $\operatorname{conv}\left(\left(e_{1}, a_{1}\right), \ldots,\left(e_{r}, a_{r}\right)\right) \cap W(\lambda)$ equals $\left(q_{e}(\lambda), q_{a}(\lambda)\right) \in \mathbb{R}^{r-1} \times \mathbb{R}^{d}$. But this is, by definition, a point in the scaled Minkowski sum - via the natural identification $\mathbb{R}^{d} \cong\left\{q_{e}(\lambda)\right\} \times \mathbb{R}^{d}=W(\lambda)$ - and every point in the Minkowski sum has this description.

The remark about the facets follows from the fact that a facet $F^{\prime}$ of $\mathcal{C}\left(\mathcal{A}_{1}, \ldots, \mathcal{A}_{r}\right)$ in $\mathbb{R}^{r-1} \times \mathbb{R}^{d}$ intersects $W(\lambda)$ if and only if it contains at least one point $\left(e_{i}, a_{i}\right)$ for each $1 \leq i \leq r$ and that a linear functional is minimized on $F^{\prime}$ over $\mathcal{C}\left(\mathcal{A}_{1}, \ldots, \mathcal{A}_{r}\right)$ if and only if its projection to $W(\lambda)$ is minimized on $F \wedge W(\lambda)$.

In order to keep the notation lean, we identify the embedding of the weighted Minkowski sum into $\mathbb{R}^{r-1} \times \mathbb{R}^{d}$ in the previous proof with the ordinary weighted Minkowski sum. The Cayley embedding $\mathcal{C}\left(\mathcal{A}_{1}, \ldots, \mathcal{A}_{r}\right)$ corresponding to the weighted Minkowski sum $\lambda_{1} \mathcal{A}_{1}+\cdots+\lambda_{r} \mathcal{A}_{r}$ will be denoted by $\left(\lambda_{1} \mathcal{A}_{1}+\cdots+\lambda_{r} \mathcal{A}_{r}\right) \vee W(\lambda)$. That is, we have

$$
\begin{aligned}
\left(\lambda_{1} \mathcal{A}_{1}+\cdots+\lambda_{r} \mathcal{A}_{r}\right) & \vee W(\lambda)=\mathcal{C}\left(\mathcal{A}_{1}, \ldots, \mathcal{A}_{r}\right), \\
\mathcal{C}\left(\mathcal{A}_{1}, \ldots, \mathcal{A}_{r}\right) & \wedge W(\lambda)=\lambda_{1} \mathcal{A}_{1}+\cdots+\lambda_{r} \mathcal{A}_{r} .
\end{aligned}
$$

Of course, this notation extends to subconfigurations as well.

The following proposition states that the "intersection" with $W(\lambda)$ induces an order-preserving map from $\omega\left(\mathcal{P}_{1} * \cdots * \mathcal{P}_{r}, \Pi_{C}\right)$ to $\omega\left(\mathcal{P}_{1} \times \ldots \times \mathcal{P}_{r}\right.$, $\left.\lambda \Pi_{M}\right)$. 
Proposition 3.4. Let $S$ be a $\Pi_{C}$-induced subdivision of $\mathcal{C}\left(\mathcal{A}_{1}, \ldots, \mathcal{A}_{r}\right)$ and

$$
S \wedge W(\lambda):=\{B \wedge W(\lambda): B \in S\} .
$$

Then

(i) $S \wedge W(\lambda)$ is a $\lambda \Pi_{M}$-induced subdivision of $\lambda_{1} \mathcal{A}_{1}+\cdots+\lambda_{r} \mathcal{A}_{r}$;

(ii) $S<S^{\prime}$ implies $(S \wedge W(\lambda))<\left(S^{\prime} \wedge W(\lambda)\right)$;

(iii) $S \wedge W(\lambda)$ is tight if $S$ is tight;

(iv) $S \wedge W(\lambda)$ is $\lambda \Pi_{M}$-coherent if $S$ is $\Pi_{C}$-coherent.

Proof. Every cell $B$ in a subdivision of a Cayley embedding is again a Cayley embedding. Therefore, by Lemma 3.2, $B \wedge W(\lambda)$ is a mixed subconfiguration in the Minkowski sum. Since for a cell in a $\Pi_{C}$-induced subdivision $S$ of $\mathcal{C}\left(\mathcal{A}_{1}, \ldots, \mathcal{A}_{r}\right)$ to be full-dimensional it must contain a point $\left(e_{i}, a_{i}\right)$ with $a_{i} \in \mathcal{A}_{i}$ for every $1 \leq i \leq r$, every cell in $S$ intersects $W(\lambda)$ in a fulldimensional subconfiguration of $\lambda_{1} \mathcal{A}_{1}+\cdots+\lambda_{r} \mathcal{A}_{r}$, thus defining a cell. This cell is clearly a projection of a face of the product $\mathcal{P}_{1} \times \cdots \times \mathcal{P}_{r}$ under $\lambda \Pi_{M}$.

The incidence structure and proper intersections are not affected by intersection with $W(\lambda)$ by Lemma 3.2. Hence, by Lemma 2.1 we get (i).

Property (ii) is obvious, (iv) is part of [21, Theorem 5.1] and (iii) follows from (ii).

The following proposition provides the inverse order-preserving map. Its proof is not difficult but nevertheless non-trivial; the extension of the polyhedral Cayley Trick from coherent to general induced subdivisions requires ingredients that are not necessary for the coherent case.

Proposition 3.5. Let $S$ be a $\lambda \Pi_{M}$-induced subdivision of $\lambda_{1} \mathcal{A}_{1}+\cdots+\lambda_{r} \mathcal{A}_{r}$ and

$$
S \vee W(\lambda):=\{B \vee W(\lambda): B \in S\} .
$$

Then

(i) $S \vee W(\lambda)$ is a $\Pi_{C}$-induced subdivision of $\mathcal{C}\left(\mathcal{A}_{1}, \ldots, \mathcal{A}_{r}\right)$;

(ii) $S<S^{\prime}$ implies $(S \vee W(\lambda))<\left(S^{\prime} \vee W(\lambda)\right)$;

(iii) $S \vee W(\lambda)$ is tight if $S$ is tight;

(iv) $S \vee W(\lambda)$ is $\Pi_{C}$-coherent if $S$ is $\lambda \Pi_{M}$-coherent.

Proof. Again, properties (ii) and (iii) are obvious, and (iv) follows from [21].

In order to prove (i), let $S$ be a $\lambda \Pi_{M}$-induced subdivision of $\lambda_{1} \mathcal{A}_{1}+\cdots+$ $\lambda_{r} \mathcal{A}_{r}$. For every cell $B$ in $S$ there is a unique cell $B \vee W(\lambda)$ in $\mathcal{C}\left(\mathcal{A}_{1}, \ldots, \mathcal{A}_{r}\right)$ with $B \vee W(\lambda) \wedge W(\lambda)=B$. Let $W^{\prime}(\lambda)=\left\{q_{e}(\lambda)\right\} \times \mathbb{R}^{p_{1}} \times \cdots \times \mathbb{R}^{p_{r}}$ be the fiber of $W(\lambda)$ under $\Pi_{C}: \mathbb{R}^{r-1} \times \mathbb{R}^{p_{1}} \times \cdots \times \mathbb{R}^{p_{r}} \rightarrow \mathbb{R}^{r-1} \times \mathbb{R}^{d}$. The cell $B$ is a projection of a face $F$ of $\mathcal{P}_{1} \times \cdots \times \mathcal{P}_{r}$, and therefore the face 
$F \vee W^{\prime}(\lambda)$ of $\mathcal{P}_{1} * \cdots * \mathcal{P}_{r}$ - recall that this equals $\mathcal{P}_{1} \times \cdots \times \mathcal{P}_{r} \vee W^{\prime}(\lambda)$ - projects to $B \vee W(\lambda)$.

For the collection of cells $S \vee W(\lambda)$ we need to show - by Lemma 2.1 that

(i) there is a point in $\operatorname{conv} \mathcal{C}\left(\mathcal{A}_{1}, \ldots, \mathcal{A}_{r}\right)$ that is contained in exactly one cell of $S \vee W(\lambda)$

(ii) adjacent cells lie on different sides of the hyperplane that supports their common facet;

(iii) for every facet $F$ of a cell $B \in S \vee W(\lambda)$ either $F$ is contained in a facet of the configuration $\mathcal{C}\left(\mathcal{A}_{1}, \ldots, \mathcal{A}_{r}\right)$ or there is another cell $B^{\prime} \in S$ containing $F$ as a facet.

First, we prove (i). Since the Minkowski sum is contained in the Cayley embedding as a section and $S$ is a subdivision of the Minkowski sum, i.e., $S$ satisfies conditions (i), (ii), and (iii), we find a point $p \in \operatorname{conv}\left(\lambda_{1} \mathcal{A}_{1}+\right.$ $\left.\cdots+\lambda_{r} \mathcal{A}_{r}\right)$ that is contained in the convex hull conv $B$ of exactly one cell $B$ of $S$. Therefore, $p$ is uniquely contained in $\operatorname{conv}(B \vee W(\lambda)) \supset \operatorname{conv} B$ where $B \vee W(\lambda) \in S \vee W(\lambda)$, which completes (i). Let $B_{1} \vee W(\lambda)$ and $B_{2} \vee W(\lambda)$ be two adjacent cells in $S \vee W(\lambda)$ with common facet $F$. Let $H$ be the hyperplane supporting $F$. We show that $B_{1} \vee W(\lambda)$ and $B_{2} \vee W(\lambda)$ lie on different sides of $H$, which proves (ii). To this end, assume $B_{1} \vee W(\lambda)$ and $B_{2} \vee W(\lambda)$ lie on the same side of $H$. Then $B_{1}=B_{1} \vee W(\lambda) \wedge W(\lambda)$ and $B_{2}=B_{2} \vee W(\lambda) \wedge W(\lambda)$ lie on the same side of $H \wedge W(\lambda)$ while $F \wedge W(\lambda)$ is the common facet of $B_{1}$ and $B_{2}$, supported by $H \cap W(\lambda)$ : contradiction to (ii) for $S$.

In order to prove (iii) we only need to observe that incidences are preserved by " $\vee W(\lambda)$ ".

See Fig. 2 for an illustration of the situation.

Remark 3.6. It is not true in general that a proper intersection of nonadjacent cells in the Minkowski sum implies a proper intersection of the corresponding cells in the Cayley embedding. See Fig. 3 for an easy example.

Propositions 3.4 and 3.5 imply Theorem 3.1. This one, in turn, has the following corollaries. The first one is straightforward.

Corollary 3.7. Weighted Minkowski sums $\sum_{i=1}^{r} \lambda_{i} A_{i}$ of a point configuration $\mathcal{A}_{1}, \ldots, \mathcal{A}_{r}$ have isomorphic posets of subdivisions for all weights $\lambda$.

In the following result we call geometric (polyhedral) subdivision of a convex polytope $\mathcal{P}$ a family of polytopes contained in $\mathcal{P}$ which cover $\mathcal{P}$ and intersect properly. If $\mathcal{P}=\operatorname{conv}(\mathcal{A})$ for a point configuration $\mathcal{A}$ then any 

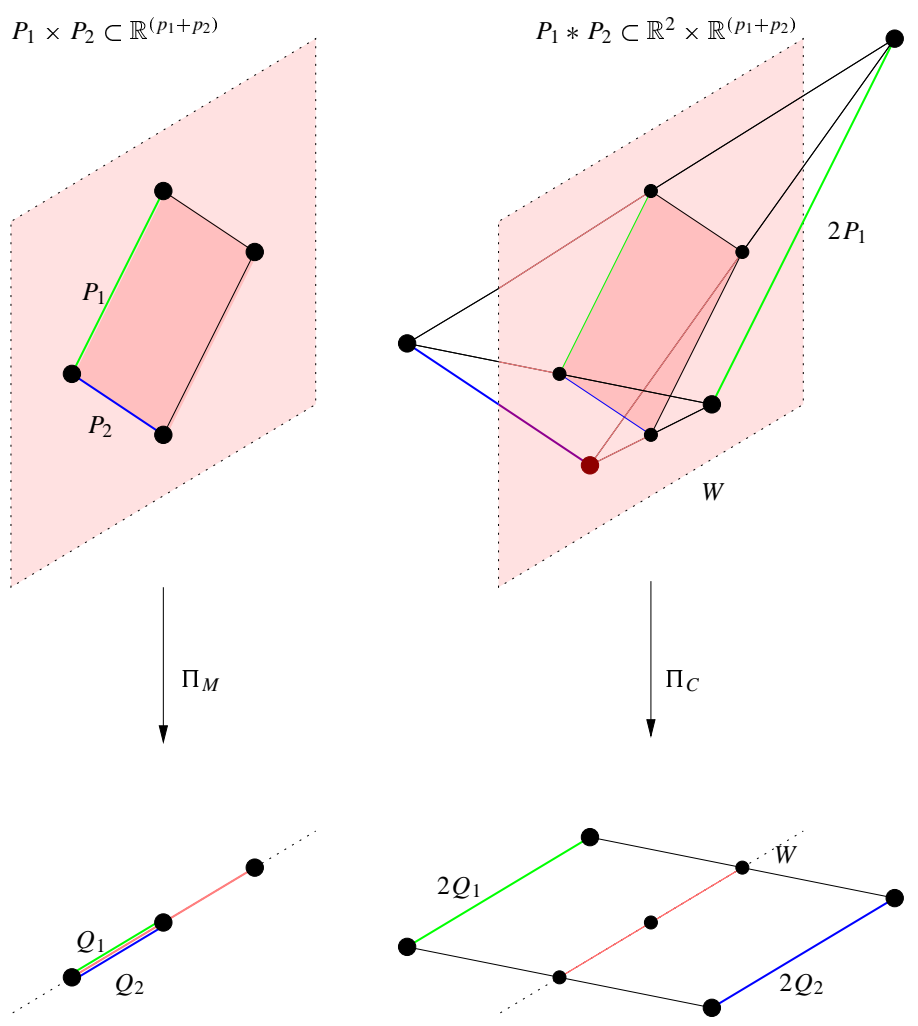

$Q_{1}+Q_{2} \subset \mathbb{R}^{q}$

$\mathcal{C}\left(Q_{1}, Q_{2}\right) \subset \mathbb{R}^{2} \times \mathbb{R}^{q}$

Fig. 2. Affine picture for $r=2$ and $P_{1}=P_{2}=[0,1]$ : product and Minkowski sum are intersections of join resp. Cayley embedding with the affine subspace $W=\left\{x_{1}=x_{2}\right.$, $\left.x_{1}+x_{2}=1\right\}$

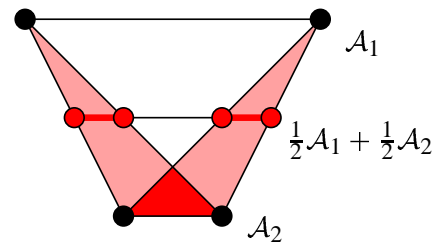

Fig. 3. Two properly intersecting cells in the Minkowski sum whose counterparts in the Cayley embedding intersect improperly

subdivision $S$ of $\mathcal{A}$ has an associated geometric subdivision $\{\operatorname{conv}(B): B \in S\}$ of $\mathcal{P}$. Reciprocally, a geometric subdivision $K$ of $\mathcal{P}$ equals $\{\operatorname{conv}(B): B \in S\}$ for some subdivision $S$ of $\mathcal{A}$ if and only if every element of $K$ has vertex set contained in $\mathcal{A}$ (but the subdivision $S$ of $\mathcal{A}$ is not unique, in general). 
Given a family $\mathcal{A}_{1}, \ldots, \mathcal{A}_{r}$ of point configurations and a geometric subdivision $K$ of the polytope $\operatorname{conv}\left(\sum_{i=1}^{r} \lambda_{i} \mathcal{A}_{i}\right)$ we say that $K$ is mixed if there is a mixed subdivision $S$ of $\sum_{i=1}^{r} \lambda_{i} \mathcal{A}_{i}$ with $K=\{\operatorname{conv}(B): B \in S\}$. A necessary condition for this to happen is that each polytope $Q$ in $K$ can be written as $\left.Q=\operatorname{conv}\left(\sum_{i=1}^{r} \lambda_{i} B_{i}\right\}\right)$ for certain subsets $B_{i} \subset \mathcal{A}_{i}, i=$ $1, \ldots, r$. But this condition is not sufficient, as the following example shows: Consider the Minkowski sum of two squares of side 1 divided into four squares of side 1 . There are 24 ways of introducing two diagonals in the four squares, and all of them provide geometric subdivisions satisfying the extra condition. But only the 8 ones with the diagonals drawn in nonadjacent squares are mixed.

Corollary 3.8. Let $\mathcal{A}_{1}, \ldots, \mathcal{A}_{r}$ be a family of point configurations, and let $K, K^{\prime}$ be geometric subdivisions of $\operatorname{conv}\left(\sum_{i=1}^{r} \lambda_{i} A_{i}\right)$. Suppose that $K$ is a refinement of $K^{\prime}$ (i.e., every cell of $K^{\prime}$ is a union of cells of $K$ ) and that $K$ is mixed. Then $K^{\prime}$ is mixed too.

Proof. An easy consequence of Theorem 3.1 is that a geometric subdivision of the geometric Minkowski sum $\operatorname{conv}\left(\sum_{i=1}^{r} \lambda_{i} A_{i}\right)$ is mixed if and only if it is the intersection of the geometric subdivision of $\operatorname{conv}\left(\mathcal{C}\left(\mathcal{A}_{1}, \ldots, \mathcal{A}_{r}\right)\right)$ associated to some subdivision of $\mathcal{C}\left(\mathcal{A}_{1}, \ldots, \mathcal{A}_{r}\right)$ with the affine subspace $W(\lambda)$.

We suppose that $K$ is the intersection with $W(\lambda)$ of a geometric subdivision $\bar{K}$ of $\operatorname{conv}\left(\mathcal{C}\left(\mathcal{A}_{1}, \ldots, \mathcal{A}_{r}\right)\right)$ and that $\bar{K}$ equals $\{\operatorname{conv}(B): B \in \bar{S}\}$ for some subdivision $\bar{S}$ of $\mathcal{C}\left(\mathcal{A}_{1}, \ldots, \mathcal{A}_{r}\right)$. Let $K=\left\{Q_{1}, \ldots, Q_{k}\right\}, K^{\prime}=$ $\left\{Q_{1}^{\prime}, \ldots, Q_{l}^{\prime}\right\}$ and $\bar{K}=\left\{\bar{Q}_{1}, \ldots, \bar{Q}_{k}\right\}$ with $Q_{i}=\overline{Q_{i}} \cap W(\lambda)$ for each $i=1, \ldots, k$.

Since $K$ refines $K^{\prime}$, for each $j=1, \ldots, l$ we can write $Q_{j}^{\prime}$ as a union of some of the $Q_{i}$ 's. We define $\overline{Q_{j}^{\prime}}$ to be the union of the corresponding $\bar{Q}_{i}$ 's, and let $\overline{K^{\prime}}:=\left\{\overline{Q_{1}^{\prime}}, \ldots, \overline{Q_{l}^{\prime}}\right\}$. We claim that $\overline{K^{\prime}}$ is a geometric subdivision of $\operatorname{conv}\left(\mathcal{C}\left(\mathcal{A}_{1}, \ldots, \mathcal{A}_{r}\right)\right)$. If this is true then it is obvious that $\overline{K^{\prime}}$ is the geometric subdivision associated to some subdivision $\overline{S^{\prime}}$ of $\mathcal{C}\left(\mathcal{A}_{1}, \ldots, \mathcal{A}_{r}\right)$ and that $K^{\prime}$ is the intersection of $\overline{K^{\prime}}$ with $W(\lambda)$, which finishes the proof.

The only non-obvious parts in the claim are that the unions $\overline{Q_{j}^{\prime}}$ are convex and that they intersect pairwise properly. We prove these two facts in the following lemma.

Lemma 3.9. Let $K$ be a geometric subdivision of the geometric Cayley embedding $\operatorname{conv}\left(\mathcal{C}\left(\mathcal{A}_{1}, \ldots, \mathcal{A}_{r}\right)\right)$. Let $Q$ and $R$ denote unions of cells in $K$.

1. If there is a weight vector $\lambda$ for which $Q \cap W(\lambda)$ is convex, then $Q$ is convex.

2. Suppose $Q$ and $R$ are convex. If there is a weight vector $\lambda_{0}$ for which $Q \cap W\left(\lambda_{0}\right)$ and $R \cap W\left(\lambda_{0}\right)$ intersect properly then $Q$ and $R$ intersect properly. 
Proof. 1. Let $Q=\left\{Q_{1}, \ldots, Q_{l}\right\}$ where the $Q_{i}$ 's are cells in the subdivision $K$. Since the $Q_{i}$ 's intersect properly, for every weight vector $\lambda$ the intersections $Q_{1} \cap W(\lambda), \ldots, Q_{l} \cap W(\lambda)$ intersect properly. Also, the polytopes $Q_{i} \cap W(\lambda)$ for different values of $\lambda$ are normally equivalent. Thus, if $Q_{i} \cap W\left(\lambda_{0}\right)$ and $Q_{j} \cap W\left(\lambda_{0}\right)$ share a face then $Q_{i} \cap W(\lambda)$ and $Q_{j} \cap W(\lambda)$ must share "the same" face for every $\lambda$ (or otherwise $Q_{i}$ and $Q_{j}$ intersect improperly). This implies that $Q \cap W\left(\lambda_{0}\right)$ and $Q \cap W(\lambda)$ are combinatorially equivalent polyhedral complexes and their boundaries are combinatorially and normally equivalent convex polytopes. Even more, their faces are labeled in the same (unique) way as intersections of faces of $Q$ with $W\left(\lambda_{0}\right)$ and $W(\lambda)$ respectively. In particular, $Q \cap W(\lambda)$ is a convex polytope for every $\lambda$.

Suppose now that $Q$ is not convex. Let $p$ and $q$ be points in $Q$ such that the segment $[p, q]$ is not contained in $Q$ and sufficiently generic so that $[p, q]$ intersects the boundary of $Q$ in the relative interior of a facet $F$ of $Q$. Let $F^{+}$be the exterior open halfspace to that facet. One of $p$ and $q$ is in $F^{+}$, suppose that it is $p$ and let $\lambda$ be the weight for which $p \in W(\lambda)$. Then, $F^{+} \cap W(\lambda)$ is the halfspace exterior to the facet $F \cap W(\lambda)$ of $Q \cap W(\lambda)$ and $p \in F^{+} \cap W(\lambda)$. This means $p \notin Q \cap W(\lambda)$, a contradiction.

2. Let $F_{0}=Q \cap R \cap W\left(\lambda_{0}\right)$ be the common face in which $Q \cap W\left(\lambda_{0}\right)$ and $R \cap W\left(\lambda_{0}\right)$ intersect. $F_{0}$ can be expressed as a union $\left(F_{1} \cup \cdots \cup F_{k}\right) \cap W\left(\lambda_{0}\right)$ where each $F_{i}$ is a face of one of the $Q_{j}$ 's in $K$ whose union equals $Q$. This expression is unique (up to reordering) if it is not redundant (i.e., if $F_{i} \cap W\left(\lambda_{0}\right)$ has the same dimension as $F_{0}$ for every $i$ ). In the same way, $F_{0}=\left(G_{1} \cup \cdots \cup G_{k^{\prime}}\right) \cap W\left(\lambda_{0}\right)$, where the $G_{i}$ 's are now faces of the cells of $K$ whose union is $R$. The fact that the $F_{i}$ 's and $G_{j}$ 's intersect properly (since they are all faces of cells of the subdivision $K$ ) together with $\left(F_{1} \cup \cdots \cup F_{k}\right) \cap W\left(\lambda_{0}\right)=\left(G_{1} \cup \cdots \cup G_{k^{\prime}}\right) \cap W\left(\lambda_{0}\right)$ for the weight $\lambda_{0}$ implies that each $F_{i}$ equals a $G_{j}$ and vice versa. Thus, $Q$ and $R$ intersect properly, in the face $F_{1} \cup \ldots \cup F_{k}=G_{1} \cup \cdots \cup G_{k^{\prime}}$.

\section{Lifting subdivisions of Lawrence polytopes and the Bohne-Dress theorem}

Throughout this section let $\mathcal{A}=\left\{a_{1}, \ldots, a_{n}\right\} \subset \mathbb{R}^{d}$ be a fixed point configuration of dimension $d$, and let $\mathcal{M}_{\mathcal{A}}$ denote the oriented matroid of affine dependences of $\mathcal{A}$, which has rank $d+1$ and ground set $\{1, \ldots, n\}$.

\subsection{Lifting subdivisions}

A lift of $\mathcal{M}_{\mathcal{A}}$ is an oriented matroid $\widehat{\mathcal{M}_{\mathcal{A}}}$ of rank $d+2$ with ground set $\{1, \ldots, n+1\}$ which satisfies $\widehat{\mathcal{M}_{\mathcal{A}}} /(n+1)=\mathcal{M}_{\mathcal{A}}$. Geometrically, a lift can 
be thought of as an embedding of $\mathcal{A}$ into $\mathbb{R}^{d+1}$ which projects "vertically" (i.e. by forgetting the last coordinate) to $\mathcal{A}$ and with the new point $n+1$ being the "point at infinity" of the vertical lines (but this picture does not take into account non-realizable lifts, or realizable lifts which do not geometrically project to $\mathcal{A}$ ).

For any $B \subset \mathcal{A}$, every lift of $\mathcal{M}_{\mathcal{A}}$ restricts to a lift of $\mathcal{M}_{B}$. Moreover, a lift of $\mathcal{M}_{\mathcal{A}}$ is fully characterized by its restrictions to circuits (minimal affinely dependent subsets) of $\mathcal{A}$. The former is trivial to check and the latter is a dualization of Proposition 7.1.4 in [6]. If $B$ is a circuit, then $\mathcal{M}_{B}$ has exactly three lifts: the "zero" lift in which $B$ lifts to a dependent set and the two lifts in which $B$ lifts to an independent set $\widehat{B}$, distinguished by the orientation they induce in the affine span of $\widehat{B}$. Hence, a lift of $\mathcal{A}$ is characterized by its circuit signature function which gives a sign $0,+$ or to each circuit.

This allows to define a natural partial order on the lifts of $\mathcal{M}_{\mathcal{A}}$, which coincides with the weak map ordering on oriented matroids with the same rank and number of elements: a lift is lower in this poset if it is "more generic" or "more uniform" see [6, Chap. 7]. More precisely, the circuit signature function of the lower lift is obtained from that of the higher by setting some zeroes to + or - .

Every lift $\widehat{\mathcal{M}_{\mathcal{A}}}$ of $\mathcal{M}_{\mathcal{A}}$ induces a subdivision $S_{\widehat{\mathcal{M}_{\mathcal{A}}}}$ of $\mathcal{A}$ as follows: a subset $\sigma \subset\{1, \ldots, n\}$ is (the set of indices of the elements of) a cell in $S$ if and only if $\sigma$ is a facet of $\widehat{\mathcal{M}_{\mathcal{A}}}$ not containing $n+1$ (a facet in an oriented matroid is the complement of a positive cocircuit [6, Chap. 9]). The subdivisions of $\mathcal{A}$ which can be obtained in this way are called lifting subdivisions. They were formally introduced in [6, Sect. 9.6], with some of the ideas coming from [4]. In the geometric picture described above the lifting subdivision is just the projection to $\mathbb{R}^{d}$ of the lower facets of the lifted point configuration. Hence, the process is a combinatorial abstraction (as well as a generalization) of the definition of regular subdivisions of $\mathcal{A}$.

The following definition and theorem, taken from [19, Sect. 5.3], provide a characterization of lifting subdivisions of $\mathcal{A}$ which does not explicitly involve the oriented matroid $\mathcal{M}$.

Definition 4.1. Let $\mathcal{A}$ be a point configuration. For each subset $B \subset \mathcal{A}$, let $S_{B}$ be a subdivision of $\mathcal{A}$. We say that the family of subdivisions $\mathcal{S}=\left\{S_{B}\right\}_{B \in \mathcal{A}}$ is consistent if for every subset $B \subset \mathcal{A}$ the following happens:

(i) For every cell $\tau \in S_{B}$ and for every $B^{\prime} \subset B, \tau \cap B^{\prime}$ is a face of a cell of $S_{B^{\prime}}$.

(ii) For every affine basis $\sigma$ of $\mathbb{R}^{d}$ contained in $B$ if $\sigma$ is contained in a cell of $S_{\sigma \cup\{b\}}$ for every $b \in B \backslash \sigma$, then $\sigma$ is contained in a cell of $S_{B}$ as well. 
We say that the family is consistent with a certain subdivision $S$ of $\mathcal{A}$ if, moreover, $S=S_{\mathcal{A}}$.

Theorem 4.2 (Santos). Let $S$ be a subdivision of a point configuration $\mathcal{A}$. Then, the following conditions are equivalent:

(i) S is a lifting subdivision.

(ii) There is a family $\mathcal{S}$ of subdivisions of the subsets of $\mathcal{M}$ which is consistent with $S$.

\subsection{Lawrence polytopes, zonotopes and the Cayley trick}

A point configuration $\mathcal{A} \subset \mathbb{R}^{d}$ of dimension $d$ with $n$ elements can be represented by an $n \times(d+1)$ matrix of rank $d+1$, whose columns are the elements of $\mathcal{A}$ with an extra coordinate equal to 1 in the last place. $\mathcal{M}_{\mathcal{A}}$ is the oriented matroid represented by the columns of this matrix, which we still denote $\mathcal{A}$. The Lawrence lifting of $\mathcal{A}$ is defined (see $[1,22]$ ) to be the point configuration corresponding to the matrix

$$
\Lambda(\mathcal{A}):=\left(\begin{array}{cc}
\mathcal{A} & \mathbf{0} \\
I & I
\end{array}\right)
$$

where $I$ is the identity matrix of size $n \times n$ and $\mathbf{0}$ the zero matrix of size $n \times(d+1)$. The $2 n$ column vectors of the matrix $\Lambda(\mathcal{A})$ affinely span a nonlinear affine hyperplane of $\mathbb{R}^{n+d+1}$, so $\Lambda(\mathcal{A})$ represents a point configuration with $2 n$ points in dimension $n+d$ which we still denote $\Lambda(\mathcal{A})$. The convex hull of this configuration is called the Lawrence polytope associated with $\mathcal{A}$. It turns out that all the points in $\Lambda(\mathcal{A})$ are vertices of this polytope.

By reordering the columns of $\Lambda(\mathcal{A})$ we see that the Lawrence polytope of $\mathcal{A}$ equals the Cayley embedding of the $n$ segments $\overline{O a_{i}} \subset \mathbb{R}^{d+1}$. I.e:

$$
\Lambda\left(a_{1}, \ldots, a_{n}\right)=\mathcal{C}\left(\overline{O a_{1}}, \ldots, \overline{O a_{n}}\right)
$$

On the other hand, the Minkowski sum of a collection of segments is a zonotope and its mixed subdivisions are usually called zonotopal tilings [24, Sect. 7.5]. We will call zonotope associated with the point configuration $\mathcal{A}$ (and denote $\mathcal{Z}(\mathcal{A})$ ) the Minkowski sum $\sum_{i=1}^{n} \overline{O a_{i}}$. Thus, the Cayley trick gives a correspondence between zonotopal tilings of the zonotope $\mathcal{Z}(\mathcal{A})$ and polyhedral subdivisions of the Lawrence polytope $\Lambda(\mathcal{A})$. 


\subsection{The Bohne-Dress theorem}

The rest of this section is devoted to prove the following theorem:

Theorem 4.3 (Bohne-Dress, Santos). Let $\mathcal{A}$ be a point configuration. The following posets are isomorphic:

(i) The poset of zonotopal tilings of $\mathcal{Z}(\mathcal{A})$.

(ii) The poset of lifts of the oriented matroid $\mathcal{M}_{\mathcal{A}}$.

(iii) The poset of subdivisions of the Lawrence polytope $\Lambda(\mathcal{A})$.

The equivalence of the first two posets is the Bohne-Dress theorem on zonotopes (see [6, Theorem 2.2.13], [24, Theorem 7.32], [18]). We provide a new proof of the Bohne-Dress theorem as follows: Our Theorem 3.1 directly implies the isomorphism between the first and last posets. The equivalence of the last two was proved in [19, Sect. 4.2] in the general case of perhaps non-realizable oriented matroids but we include here a shorter proof. The essential new feature of our proof is that we use Santos' characterization of lifting subdivisions (Theorem 4.2) to prove part (ii) of the following statement, while in [19] the same thing is proved directly.

Proposition 4.4. Let $\mathcal{A}$ be a point configuration with oriented matroid $\mathcal{M}_{\mathcal{A}}$, and let $\Lambda(\mathcal{A})$ be the associated Lawrence polytope, with oriented matroid $\mathcal{M}_{\Lambda(\mathcal{A})}$. Then:

(i) Two different lifts of $\mathcal{M}_{\Lambda(\mathcal{A})}$ produce different associated lifting subdivisions.

(ii) Every subdivision of $\Lambda(\mathcal{M})$ is a lifting subdivision.

(iii) The poset of lifts of $\mathcal{M}_{\Lambda(\mathcal{M})}$ and the poset of lifts of $\mathcal{M}_{\mathcal{A}}$ are isomorphic.

Thus, the poset of lifts of $\mathcal{M}_{\mathcal{A}}$ and the poset of subdivisions of $\Lambda(\mathcal{A})$ are isomorphic.

Proof. Throughout the proof we will denote by $b_{1}, \ldots, b_{n}, e_{1}, \ldots, e_{n}$ the vertices of the Lawrence polytope, that is to say the columns of the matrix

$$
\Lambda(\mathcal{A}):=\left(\begin{array}{cc}
\mathcal{A} & \mathbf{0} \\
I & I
\end{array}\right)
$$

The following are some other very special properties of $\Lambda(\mathcal{A})$. Let $C=\left(C^{+}, C^{-}\right)$be a circuit of $\Lambda(\mathcal{A})$. This means that $C^{-} \cup C^{+}$is a minimal dependent set and that $C^{-}$and $C^{+}$are, respectively, the sets of points with positive and negative coefficient in the unique (modulo a scalar) dependence equation involving them. The structure of the matrix $\Lambda(\mathcal{A})$ clearly implies that whenever an element $b_{i}$ or $e_{i}$ is in $C^{+}$the companion $e_{i}$ or $b_{i}$ is in $C^{-}$ and vice versa. In other words, the support of every circuit has the form 
$\left\{b_{i}: i \in J\right\} \cup\left\{e_{i}: i \in J\right\}$, for some $J \subset\{1, \ldots, n\}$. On the other hand, any subset of that form is a face of $\Lambda(\mathcal{A})$.

If $B$ is now an arbitrary subset of the vertices of $\Lambda(\mathcal{A})$, let $B_{0}=$ $\left\{b_{i} \in B: e_{i} \in B\right\} \cup\left\{e_{i} \in B: b_{i} \in B\right\}$. Every element $p \in B \backslash B_{0}$ is a coloop in $B$. In other words, for every subset $B$ of the vertices of $\Lambda(\mathcal{A})$, $\operatorname{conv}(B)$ is an iterated cone over the face $\operatorname{conv}\left(B_{0}\right)$ of $\Lambda(\mathcal{A})$. These facts will be crucial in the proof of the three statements:

(i) The circuit signature functions of two different lifts will necessarily give different sign to a certain circuit $C$ of $\Lambda(\mathcal{A})$. But this implies that the associated lifting subdivisions are different, since they are different in the face of $\Lambda(\mathcal{A})$ spanned by the support of that circuit. (The three lifts of a circuit induce, respectively, the trivial subdivision and the two unique triangulations of the circuit).

(ii) Since every subset $B$ of the vertices of $\Lambda(\mathcal{A})$ is an iterated cone over a face $\operatorname{conv}\left(B_{0}\right)$, a subdivision $S$ of $\Lambda(\mathcal{M})$ is consistent with only one subdivision of $B$ : the cone over the subdivision of the face $\operatorname{conv}\left(B_{0}\right)$ induced by $S$. Let $\left\{S_{B}\right\}_{B \subset \Lambda(\mathcal{A})}$ denote the family of subdivisions so obtained. The first condition of consistency is trivially satisfied by this family. For proving the second one we will use induction on the dimension of the subset $B$ involved.

Let $\sigma$ be a basis contained in $B$ such that for every $b \in B \backslash \sigma$ we have that $\sigma$ is in a cell of the subdivision $S_{\sigma \cup\{b\}}$. Since $\sigma$ is full-dimensional, it must contain at least one of each pair of vertices $b_{i}$ and $e_{i}$ of $\Lambda(\mathcal{A})$, for every $i \in\{1, \ldots, n\}$. On the other hand, since the case $\sigma=\Lambda(\mathcal{A})$ is trivial, $\sigma$ contains an element $e_{i}$ or $b_{i}$ whose companion $e_{i}$ or $b_{i}$ is not in $\sigma$. Let $a$ be such an element, and let us denote its companion by $\bar{a}$.

Since $\{a, \bar{a}\}$ is the complement of the set of vertices of a facet of $\Lambda(\mathcal{A})$, by induction on the dimension we assume that $\sigma \backslash\{a\}$ lies in a cell of $S_{B \backslash\{a, \bar{a}\}}$. If $\bar{a} \notin B$ this implies that $\sigma$ lies in a cell of $S_{B}$. If $\bar{a} \in B$ we still can conclude that either $\sigma$ or $\sigma \backslash a \cup\{\bar{a}\}$ lie in a cell of $S_{B}$. So suppose that the second happens, and let $\tau$ be that cell. We will proof that $a \in \tau$ as well.

Consider the corank 1 subconfiguration $B^{\prime}=\sigma \cup\{\bar{a}\}$ of $B$. By the first condition of consistency, $\tau \cap B^{\prime}$ is a face of a cell in $S_{B^{\prime}}$. On the other hand, since $B^{\prime}$ is of the form $\sigma \cup\{b\}, \sigma$ lies in a cell of $S_{B^{\prime}}$ by hypothesis. Thus, both $B^{\prime} \backslash\{\bar{a}\}=\sigma$ and $B^{\prime} \backslash\{a\} \subset \tau \cap B^{\prime}$ lie in cells of $S_{B^{\prime}}$. Since $B^{\prime} \backslash\{a, \bar{a}\}$ is a face of $B^{\prime}$, this implies that $S_{B^{\prime}}$ is the trivial subdivision. Finally, since $\tau \cap B^{\prime}$ is full dimensional because it contains $\sigma \backslash\{a\} \cup\{\bar{a}\}, \tau \cap B^{\prime}$ is a cell of $S_{B}^{\prime}$ and, thus, $a \in \tau$, as we wanted to prove.

(iii) Let $\mathcal{A}^{*}$ be a Gale transform of $\mathcal{A}$, represented as a matrix of size $n \times(n-d-1)$ whose row space $\operatorname{row}\left(\mathcal{A}^{*}\right)$ is an orthogonal complement of $\operatorname{row}(\mathcal{A})$. Then, the matrix $\left(\mathcal{A}^{*},-\mathcal{A}^{*}\right)$ of size $2 n \times(n-d-1)$ represents a Gale transform of $\Lambda(\mathcal{A})$. In other words, the oriented matroid dual to 
$\mathcal{M}_{\Lambda(\mathcal{A})}$ is obtained from the dual of $\mathcal{M}_{\mathcal{A}}$ by adjoining an antiparallel element to every element. Then, it is trivial that the two duals have the same posets of extensions (for example, via the topological representation theorem of oriented matroids; also via Las Vergnas' characterization of extensions by cocircuit signature functions. See [6]). Since lifts of an oriented matroid are duals to extensions of its dual, part (iii) is proved.

Once we have proved parts (i), (ii), and (iii) we have a bijection between the two posets we are interested in. That this bijection is a poset isomorphism is trivial.

\section{References}

1. M. Bayer, B. Sturmfels: Lawrence polytopes. Can. J. Math. 42, 62-79 (1990)

2. L.J. Billera, B. Sturmfels: Fiber polytopes. Ann. Math. 13, 527-549 (1992)

3. L.J. Billera, P. Filliman, B. Sturmfels: Constructions and complexity of secondary polytopes. Adv. Math. 83, 155-179 (1990)

4. L.J. Billera, B.S. Munson: Triangulations of oriented matroids and convex polytopes. SIAM J. Alg. Disc. Methods 5, 515-525 (1984)

5. J. Bohne: Eine kombinatorische Analyse zonotopaler Raumaufteilungen. Dissertation, Fachbereich Mathematik, Universität Bielefeld, $100 \mathrm{pp}$

6. A. Björner, M. Las Vergnas, B. Sturmfels, N. White, G. Ziegler: Oriented Matroids. Cambridge: Cambridge University Press 1992

7. J. Canny, I. Emiris: Efficient incremental algorithms for the sparse resultant and the mixed volume. J. Symbolic Computation 20, 117-149 (1995)

8. J.A. De Loera, S. Hosten, F. Santos, B. Sturmfels: The polytope of all triangulations of a point configuration. Doc. Math. J. DMV 1, 103-119 (1996)

9. I.M. Gel'fand, M.M. Kapranov, A.V. Zelevinsky: Multidimensional Determinants, Discriminants and Resultants. Boston: Birkhäuser 1994

10. B. Huber: Solving Sparse Polynomial Systems. Ph.D. Thesis, Cornell University, 1996

11. B. Huber, B. Sturmfels: A polyhedral method for solving sparse polynomial systems. Math. Comp. 64, 1541-1555 (1995)

12. M. Las Vergnas: Extensions ponctuelles d'une géométrie combinatoire orienté. In: Problémes combinatoires et théorie des graphes (Actes Coll. Orsay 1976) 265-270. Colloques Internationaux 260, C.N.R.S, Paris

13. T. Michiels, J. Verschelde: Enumerating regular mixed-cell configurations. Disc. Comput. Geom. 21, 569-579 (1999)

14. P. Pedersen, B. Sturmfels: Product formulas for resultants and Chow forms. Math. Z. 214, 377-396 (1993)

15. J. Rambau: Triangulations of cyclic polytopes and higher Bruhat orders. Mathematika 44, 162-194 (1997)

16. J. Rambau, G.M. Ziegler: Projections of polytopes and the Generalized Baues Conjecture. Disc. Comput. Geom. 16, 215-237 (1996)

17. V. Reiner: The generalized Baues problem. In: New Perspectives in Algebraic Combinatorics. L. Billera, A. Björner, C. Greene, R.E. Simion, P. Stanley (eds.). MSRI Book Series 38, 293-336. Cambridge: Cambridge University Press 1999

18. J. Richter-Gebert, G. Ziegler: Zonotopal tilings and the Bohne-Dress theorem. In: Jerusalem Combinatorics'93. H. Barcelo, G. Kalai (eds.) 211-232, Contemporary Mathematics 178, Amer. Math. Soc. 1994

19. F. Santos: Triangulations of oriented matroids, preprint 1997, 78 pages. In: Memoirs of the AMS (accepted for publication)

20. F. Santos: Applications of the polyhedral Cayley Trick to triangulations of polytopes. (in preparation) 
21. B. Sturmfels: On the Newton polytope of the resultant. J. Algebr. Combinatorics 3 , 207-236 (1994)

22. B. Sturmfels: Gröbner bases and convex polytopes. University Lecture Series 8, American Mathematical Society, Providence, 1995

23. J. Verschelde, K. Gatermann, R. Cools: Mixed-volume computation by dynamic lifting applied to polynomial system solving. Disc. Comput. Geom. 16, 69-112 (1996)

24. G.M. Ziegler: Lectures on Polytopes. Graduate Texts in Mathematics 152. New York: Springer 1995 